\title{
Development of the Functional Connectome Topology in Adolescence: evidence from Topological Data Analysis
}

Zeus Gracia-Tabuenca ${ }^{1}$, Juan Carlos Díaz-Patiño², Isaac Arelio², Martha Beatriz Moreno $^{1}$, Fernando A. Barrios ${ }^{1}$, Sarael Alcauter ${ }^{1, *}$

${ }^{1}$ Universidad Nacional Autónoma de México, Instituto de Neurobiología, Querétaro, Mexico.

${ }^{2}$ Universidad Nacional Autónoma de México, Instituto de Matemáticas, Querétaro, Mexico.

* corresponding author: alcauter@inb.unam.mx

\section{Abstract}

Adolescence is a crucial developmental period in terms of behavior and mental health. Therefore, understanding how the brain develops during this stage is a fundamental challenge for neuroscience. Recent studies have modelled the brain as a network or connectome, mainly applying measures from graph theory, showing a change in its functional organization such as an increase in its segregation and integration. Topological Data Analysis (TDA) complements such modelling by extracting high-dimensional features across the whole range of connectivity values, instead of exploring a fixed set of connections. This study enquiries into the developmental trajectories of such properties using a longitudinal sample of typically developing participants ( $\mathrm{N}=98 ; 53 / 45 \mathrm{~F} / \mathrm{M}$; 6.7-18.1 years), applying TDA into their functional connectomes. In addition, we explore the effect of puberty on the individual developmental trajectories. Results showed that compared to random networks, the adolescent brain is more segregated at the global level, but more densely connected at the local level. Furthermore, developmental effects showed nonlinear trajectories for the integration of the whole brain and fronto-parietal 
networks, with an inflection point and increasing trajectories after puberty onset. These results add to the insights in the development of the functional organization of the adolescent.

\section{Significance Statement}

Topological Data Analysis may be used to explore the topology of the brain along the whole range of connectivity values instead of selecting only a fixed set of connectivity thresholds. Here, we explored some properties of the topology of the brain functional connectome, and how they develop in adolescence. First, we show that developmental trajectories are nonlinear and better explained by the puberty status than chronological age, with an inflection point around the puberty onset. The greatest effect is the increase in functional integration for the whole brain, and particularly for the Fronto-Parietal Network when exploring functional subnetworks.

\section{Introduction}

Adolescence is a critical development period with substantial impact on body and behavior. Particularly, the brain undergoes structural and functional changes that are influenced by pubertal hormones (Vijayakumar et al., 2018; Laube et al., 2020). Moreover, these changes occur along with a consolidation of cognitive and executive performance (Baum et al., 2017; Chai et al., 2017).

These insights had been addressed modelling the brain as a complex network of interacting components, either at task or rest conditions (Biswal et al., 1995; Smith et al., 2009). In this framework, the functional connectome is described by its system properties in biologically plausible terms, mainly using measures from graph theory (Rubinov and Sporns, 2010). Nevertheless, other methods had recently been applied 
to address high-dimensional data such as Topological Data Analysis (TDA) (Sizemore et al., 2018; Expert et al., 2019). TDA models the connectome as a topological space and characterizes its interaction patterns as geometric features, allowing it to simplify complex structures at different scales (Giusti et al., 2016; Santos et al., 2019; Centeno et al., 2021). In particular, TDA applied to functional connectomes is not affected by the potential biases of connectivity thresholding nor brain segmentation (Lee et al., 2012; Gracia-Tabuenca et al., 2020).

In terms of functional organization of the brain, previous cross-sectional studies have shown that the adolescent period is characterized by an increase of the modularity and specialization (Fair et al., 2009; Satterthwaite et al., 2013a; Gu et al., 2015), with prominent effects in frontal and parietal systems, along with executive performance (Marek et al., 2015; Gracia-Tabuenca et al., 2021). However, as far as we are concerned, TDA in human connectomes have mainly been applied into neuropsychiatric disorders (Lee et al., 2012, 2017; Gracia-Tabuenca et al., 2020; Li et al., 2020), but not to characterize the typical development. There is still a huge degree of incertitude in this field due to the great variability between samples, sexes, and cultures (Sawyer et al., 2018), with special emphasis in the fact that some individuals have faster or slower pubertal development even when they have the same chronological age (Blakemore et al., 2010; Vijayakumar et al., 2018). To this regard, longitudinal trajectories and pubertal markers are highly valuable to describe adolescent development.

Therefore, this study focuses on characterizing the development of the functional connectome in the adolescent period applying TDA into a longitudinal sample of typically developing subjects. In addition, the effect of pubertal status and linear vs. nonlinear trends are tested as well. 


\section{Methods}

\subsection{Sample}

A general invitation was sent to local schools describing the study protocols and the inclusion/exclusion criteria. Inclusion criteria consisted of a full-term gestation (more or equal than 37 weeks). Exclusion criteria included academic year repetition and any neurological or psychiatric disorder identified with the MINI semi-structured interview. Signed informed consent for parents and verbal assent for minors was required. The study protocols followed the ethical principles of the Declaration of Helsinki and were approved by the Institutional Ethics Board.

The sample consisted of 98 typically developing participants (53 females, 45 males; age range: 6.7 - 18.1 years old). From those, 41 returned for a second session, and 16 for a third. Follow-ups occurred after 5 years and the second after 2 years, respectively.

\subsection{Pubertal status assessment}

Participants fulfilled the Pubertal Development Scale (PDS; Petersen et al., 1988). PDS averages the response of five self-reported questions about growth spurt in height, pubic hair, and skin change for both sexes; plus breast growth and menarche for females and facial hair growth and voice change for males. Responses are absence (1), first signs (2), evident (3), and finished (4) pubertal spurt. Those participants under 10 years old were set to PDS level 1, following similar values in previous studies (Hibberd et al., 2015; van Duijvenvoorde et al., 2019). In addition, 8 missing values (4 females) were estimated via Generalized Additive Mixed Model 
(GAMM) with age-sex interaction locally estimated scatterplot smoothing (LOESS) curves.

\subsection{Imaging}

For each session, participants underwent an MRI protocol including a whole-brain fMRI sequence plus high-resolution T1-weighted images for anatomical reference. After five 'dummy' volumes for scan stabilization, a total of $150 \mathrm{fMRI}$ volumes were obtained using a gradient recalled $\mathrm{T}^{*}$ echo-planar imaging sequence (TR/TE = $2000 / 40 \mathrm{~ms}$, voxel size $4 \times 4 \times 4 \mathrm{~mm}^{3}$ ). Participants were instructed to lay down, close their eyes, and not to fall asleep. In order to ease participants to remain awake, the $\mathrm{fMRI}$ scan was applied at the beginning of the MRI session and always in the morning. T1 images were obtained using a 3D spoiled gradient recalled (SPGR) acquisition $\left(\mathrm{TR} / \mathrm{TE}=8.1 / 3.2 \mathrm{~ms}\right.$, flip angle $=12.0$, voxel size $\left.1 \times 1 \times 1 \mathrm{~mm}^{3}\right)$. All brain imaging was acquired with a 3T MR GE750 Discovery scanner (General Electric, Waukesha, WI), using an 8-channel-array head coil. However, 20 sessions were acquired with a 32-channel coil, thus a covariate was included in the subsequent analyses.

\subsection{Preprocessing}

Structural T1 volumes were denoised with non-local means (Manjón et al., 2010) and N4 bias field correction (Tustison et al., 2010). fMRI datasets were preprocessed using FSL v.5.0.6 (Jenkinson et al., 2012; RRID:SCR_002823). Preprocessing steps included slice timing, head motion correction, brain extraction, intensity 
normalization, confound regression, spatial normalization, and 0.01-0.08 Hz bandpass filtering.

Considering that the pediatric population tends to move more inside the scanner (Satterthwaite et al., 2012), we implemented a strident strategy of confounding variables regression (Satterthwaite et al., 2013b). 36 parameters were regressed out from the $\mathrm{fMRI}$ time series, including the six head-motion estimated parameters plus the average time series of the global signal, white matter, and cerebrospinal fluid. The derivatives of these nine variables were also added, and the quadratic terms of those eighteen. Additionally, the volumes with a framewise displacement (FD-RMS; Jenkinson et al., 2002) greater than $0.25 \mathrm{~mm}$ ("spikes") were included as confounds as well. This approach overpowers other widely used motion-mitigation methods (Ciric et al., 2017; Parkes et al., 2018; Graff et al., 2020). Eighteen sessions with less than four minutes without spike-volumes were discarded (Satterthwaite et al., 2013b; Parkes et al., 2018), therefore, the final sample consisted of 89 participants (39 male, age range: 6.7-18.1 y.o.), of whom 37 and 11 had two and three longitudinal sessions, respectively.

In addition, $\mathrm{fMRI}$ datasets were co-registered to their $\mathrm{T} 1$ volume with six degrees of freedom, and then warped twice using nonlinear SyN transformation (Avants et al., 2008; RRID:SCR_004757) to a pediatric template (NIHPD4.5-18.5; Fonov et al., 2011) and then to the MNI-152 standard template. 


\subsection{Functional Connectomes}

Brain networks were defined based on 264 regions of interest (ROls) as nodes (Power et al., 2011). Pairwise edges were calculated through Pearson's correlation between the average fMRI preprocessed signal of every pair of ROls.

These ROls consist of $5 \mathrm{~mm}$-radius spheres with high consistency in task and rest tested in large fMRI databases (Power et al., 2011). Moreover, this set of ROls can be grouped in thirteen functional networks. This segmentation has been applied in numerous pediatric studies (Satterthwaite et al., 2013a; Gu et al., 2015; Marek et al., 2015; Chai et al., 2017; Ciric et al., 2017; Gracia-Tabuenca 2020, 2021).

\subsection{Topological Data Analysis}

The functional connectome can be modeled as a topological space by the means of the Rips complex, defined as $\operatorname{Rips}(F, \square)$. F stands for the set of nodes (same as the connectome nodes) and $\square$ stands for the filtration value, which is a positive number that indicates which nodes of $F$ with lower distance than it are connected. Then, the set of connected nodes of the Rips complex varies as a function of $\square$. Additionally, algebraic properties can be extracted from the Rips complexes, the so-called Betti numbers. Specifically, Betti numbers of order zero or Betti-0 $\left(\mathrm{B}_{0}\right)$ accounts for the number of components (i.e., the sum of groups of connected and isolated nodes), Betti-1 $\left(B_{1}\right)$ accounts for the number of "holes" in the two-dimensional space between connected nodes (Figure 1), and so on (for extensive review on TDA, we suggest Edelsbrunner et al., 2000; Sizemore et al., 2019). 

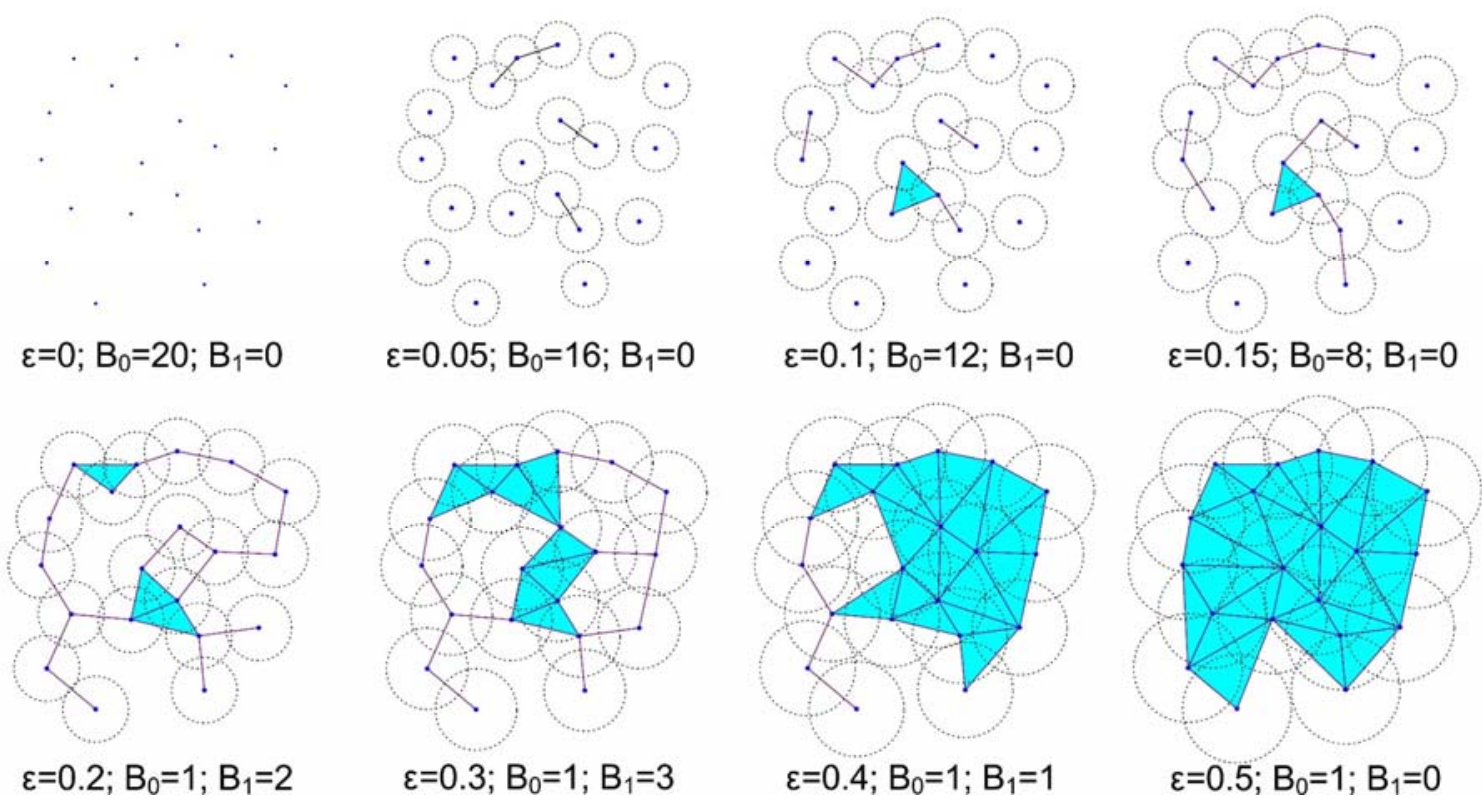

Figure 1. Betti-0 and Betti-1. Set of 20 nodes, eight filtration values $\varepsilon$, represented as the circle diameter and their corresponding Betti-0 $\left(B_{0}\right)$ and Betti-1 $\left(B_{1}\right)$. At $\varepsilon=0$ the number of components (Betti-0) is equal to the number of nodes, even without links there are no holes. As the filtration value increases, the number of components (Betti-0) reduces while the number of holes (Betti-1) increases. A hole is a polygon with four or more sides, by definition a triangle is not a hole and if this is the case, we put a triangular surface (light blue). Eventually it will reach a single component containing all nodes and the number of holes becomes zero. Blue triangles are not holes as they are surfaces between triads of nodes.

In this study we focused exclusively on $B_{0}$ and $B_{1}$. As $\square$ increases, the number of isolated nodes decreases in favour of the connected nodes, that is the $B_{0}$ decreases and eventually will reach a single component where every node of $F$ is connected (Figure 1). In contrast, at low values of $\square$ there are no holes in the connected pattern of the topological space because there are not enough connections to build them. Similarly, at high values of $\square$ the holes are "filled" because all pairwise connections 
within the component are accomplished. These holes represent serially distributed connections of nodes without shortcuts between them, while a filled hole means that those nodes are densely connected between them (Sizemore et al., 2018). Therefore, the greater amount of holes (i.e., $B_{1}$ ) is reached at intermediate values of $\square$. Both processes can be characterized by Betti curves as a function of $\square$ (Figures 2 and 3).

The distance between nodes was set as one minus their corresponding Pearson's correlation (i.e., their functional connectivity edge), following Lee et al. (2012): $d\left(x_{i}, x_{j}\right)=1-r\left(x_{i}, x_{j}\right)$. Being $r$ the Pearson's correlation between nodes $x_{i}$ and $\mathrm{x}_{\mathrm{j}}$. $\mathrm{B}_{0}$ and $\mathrm{B}_{1}$ curves were computed using the TDA R-package (Fasy et al., 2014), and were summarized by means of the area under the curve (AUC). The AUC accounts for the overall process of the Betti numbers along all possible values of $\square$. Low scores of $B_{0}$-AUC can be interpreted as a fast transition to the single component, while higher scores imply a more segregated configuration of the brain network. Meanwhile, low scores of $B_{1}-A \cup C$ mean that distributed connected components rapidly bind to one another, and higher scores imply an increase in the number of holes within the network (i.e., a more distributed connectivity structure).

Furthermore, to discard that the observed results can be obtained by chance, a null distribution of Betti curves was generated by bootstrapping 1000 connectomes extracted from the original sample whose edges were randomly rewired (Giusti et al., 2015; Gracia-Tabuenca et al., 2020). 


\subsection{Developmental trajectories}

Developmental effects were tested using linear mixed-effects (LME) and nonlinear generalized additive mixed models (GAMM). Six models were applied: two LME for age and age-sex interaction, two GAMM fitting smooth splines for age and age-bysex. However, given that PDS is an ordinal and not a continous scale, nonparametric locally estimated scatterplot smoothing (LOESS) terms for PDS and PDS-sex interaction were included in the two remaining GAMMs. Every model included random-effects for the intercept plus average head motion (FD-RMS) and coil as confounds. Random-effects were estimated via maximum likelihood. Models were implemented using $\mathrm{R}$ libraries: LME via Ime4 (Bates et al., 2007; RRID:SCR_015654), GAMM with splines via gamm4 (Wood et al., 2017), GAMM with LOESS via gam/ss (Stasinopoulos and Rigby, 2007). Model selection was set by the lowest Akaike Information Criterion (AIC; Akaike, 1974). The AIC evals a model by the tradeoff between its complexity and its goodness of fit. That is, the subtraction between the number of parameters $(k)$ and the log-likelihood function $(\ln L)$ by a factor of two (i.e., $\mathrm{AIC}=2 k-2 \ln L)$.

In addition, developmental effects within the model were tested using a "dropterm" Likelihood Ratio Test (LRT) approach. The LRT contrasts the full model in relation to a null model without the term of interest. Also, LRT was applied for the thirteen functional networks of the Power et al. (2011) segmentation, where their corresponding significance was corrected for multiple testing using a False Discovery Rate (FDR) q < 0.05 (Benjamini and Hochberg, 1995). 


\subsection{Code accessibility}

All preprocessed data and the code described in this study are freely available online at \#\#\#. Also, the code is available as Extended Data 1. Present results were computed with an Intel Core i7-4790 CPU @ 3.60 $\square \mathrm{GHz} \square \times \square 8$ with Ubuntu 18.04.3 LTS 64-bit.

\section{Results}

The sample intercept $B_{0}$ curve, that is, the representative curve for the whole sample, showed an inverse sigmoid pattern with a slower transition to the single component compared to the permuted data (Figure 2). On the other hand, the $B_{1}$ curve shows a bell-shape with a maximum of 104.69 "holes" at 0.432 filtration value, while the permuted data shows a maximum of 183.92 at $\square=0.438$ (Figure 3). 


\section{Betti-0 sample intercept}



Filtration Value

Figure 2. Sample intercept $B_{0}$ curve (with $95 \%$ confidence interval) in blue. Average of 1000 bootstrapped connectomes with random edge-rewiring $B_{0}$ curve (with $95 \%$ confidence interval) in gray. 


\section{Betti-1 sample intercept}



Filtration Value

Figure 3. Sample intercept $B_{1}$ curve (with $95 \%$ confidence interval) in blue. Average of 1000 bootstrapped connectomes with random edge-rewiring $B_{1}$ curve (with $95 \%$ confidence interval) in gray.

Regarding model selection for the developmental effects, the GAMM for the PDS showed the lowest AIC for $B_{0}-A U C$ (834.83) and $B_{1}$-AUC (615.71) (Table 1).

Table 1. Akaike Information Criterion (AIC) for Betti-0 ( $\left.B_{0}\right)$ and Betti-1 $\left(B_{1}\right)$ areas under the curve (AUC) at every developmental model: linear mixed-effects models for age (LME-Age) and age-sex interaction (LME-Age.Sex), generalized additive mixed models with smooth splines for age (GAMM-Age) and age-by-sex (GAMMAge.Sex), and with LOESS terms for PDS (GAMM-PDS) and PDS-sex interaction (GAMM-PDS.Sex). The smallest values in bold. 


\begin{tabular}{|c|c|c|c|c|c|c|}
\hline & LME-Age & $\begin{array}{l}\text { LME- } \\
\text { Age.Sex }\end{array}$ & GAMM-Age & $\begin{array}{l}\text { GAMM- } \\
\text { Age.Sex }\end{array}$ & $\begin{array}{l}\text { GAMM- } \\
\text { PDS }\end{array}$ & $\begin{array}{l}\text { GAMM- } \\
\text { PDS.Sex }\end{array}$ \\
\hline$B_{0}-A \cup C$ & $\begin{array}{l}863 \\
.05\end{array}$ & $\begin{array}{l}864.8 \\
6\end{array}$ & $\begin{array}{l}861.3 \\
3\end{array}$ & $\begin{array}{l}862.1 \\
9\end{array}$ & $\begin{array}{l}83 \\
4 . \\
83\end{array}$ & $\begin{array}{l}837 \\
.84\end{array}$ \\
\hline $\mathrm{B}_{1}-\mathrm{AUC}$ & $\begin{array}{l}627 \\
.61\end{array}$ & $\begin{array}{l}631.3 \\
3\end{array}$ & $\begin{array}{l}627.4 \\
9\end{array}$ & 630.2 & $\begin{array}{l}61 \\
5 . \\
71\end{array}$ & $\begin{array}{l}622 \\
.15\end{array}$ \\
\hline
\end{tabular}

The $B_{0}$ - and $B_{1}-A U C$ trends along the PDS show an initial increase from level 1 to 2 , followed by a soft decline after that (Figure 4). However, only the PDS term was significant for the $B_{0}-A U C(L R T=12.18 ; D O F=2.11 ; p=0.0026)$. Furthermore, the random-effects term was only significant for the $B_{0}$-AUC $(L R T=79.86$; DOF $=30.96$; $p=3.38 e-06)$ 


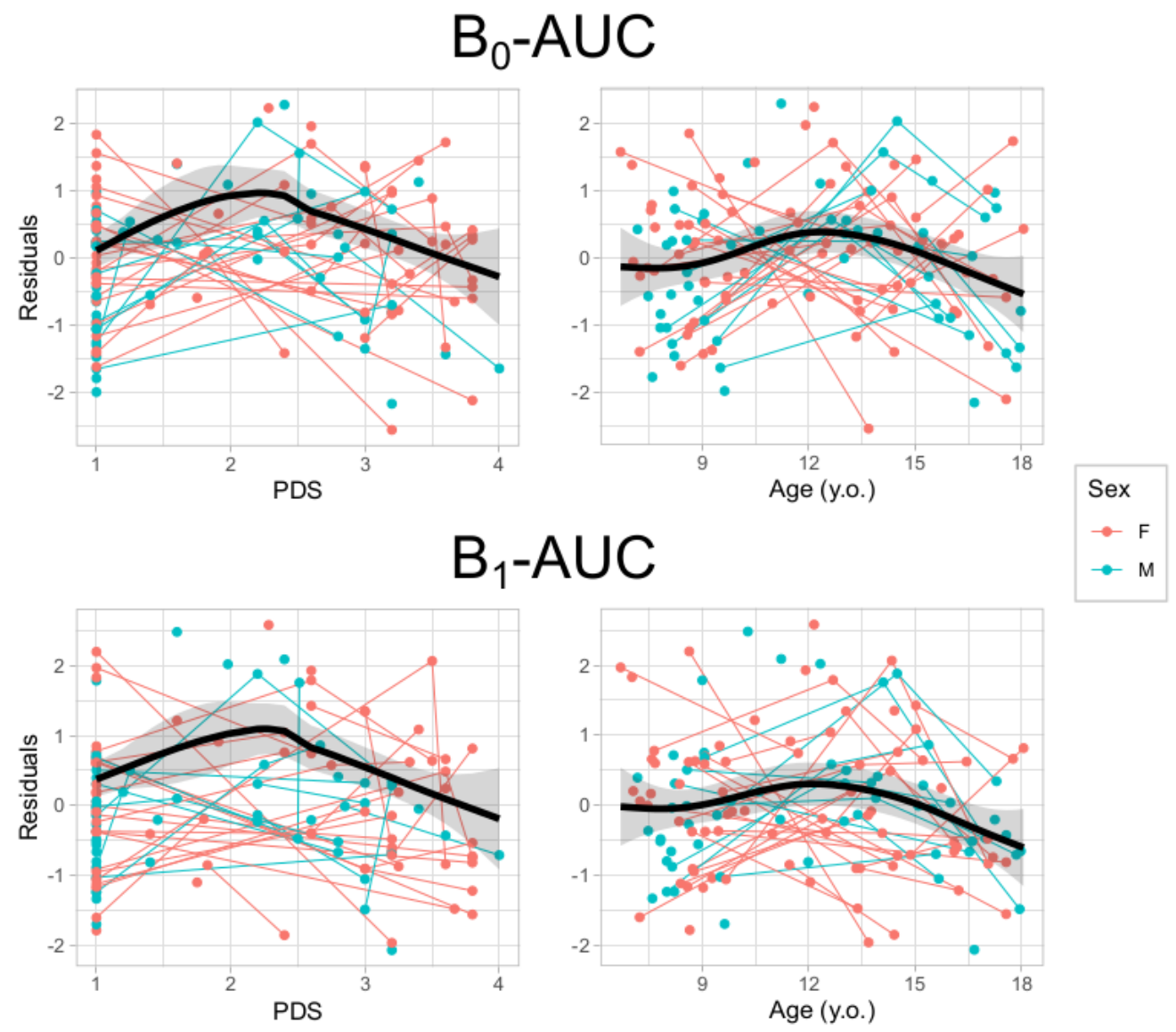

Figure 4. Scatter-plots of the GAMM PDS-LOESS (left) and age-spline (right) models for the $B_{0}$ (top) and $B_{1}$ (bottom) area under de curve (AUC) residuals (after regressing out in-scanner motion and head-coil) in relation to the pubertal scale (PDS) or age. Thin lines represent individual trajectories; thick black lines represent the sample curve (with 95\% confidence-interval shadow).

Concerning the developmental effects at the functional network level, PDS term showed strong effects in the Fronto-Parietal (FPN) and moderate effects in the Auditory (AUD), sensorimotor-hand (SMH), and subcortical (SUB) networks for the $\mathrm{B}_{0}-\mathrm{AUC}$ (Figure 5). Only the FPN had a significant effect after FDR correction (LRT = 26.18; DOF $=7.21 ; p=5.52 \mathrm{e}-04)$, which shows a nonlinear trend similar to that for 
the whole brain network (Figure 5). No effects (even uncorrected) were found for the $B_{1}-A U C$.

$\mathbf{L}$
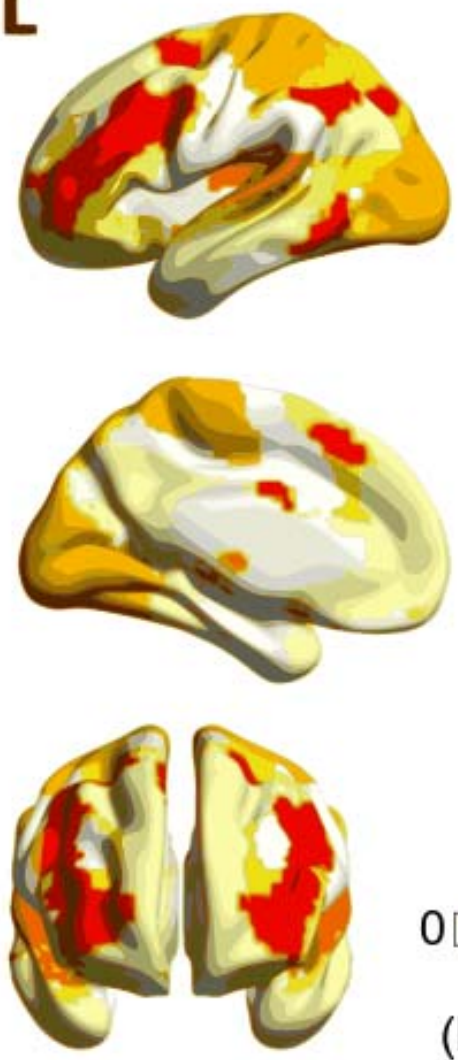
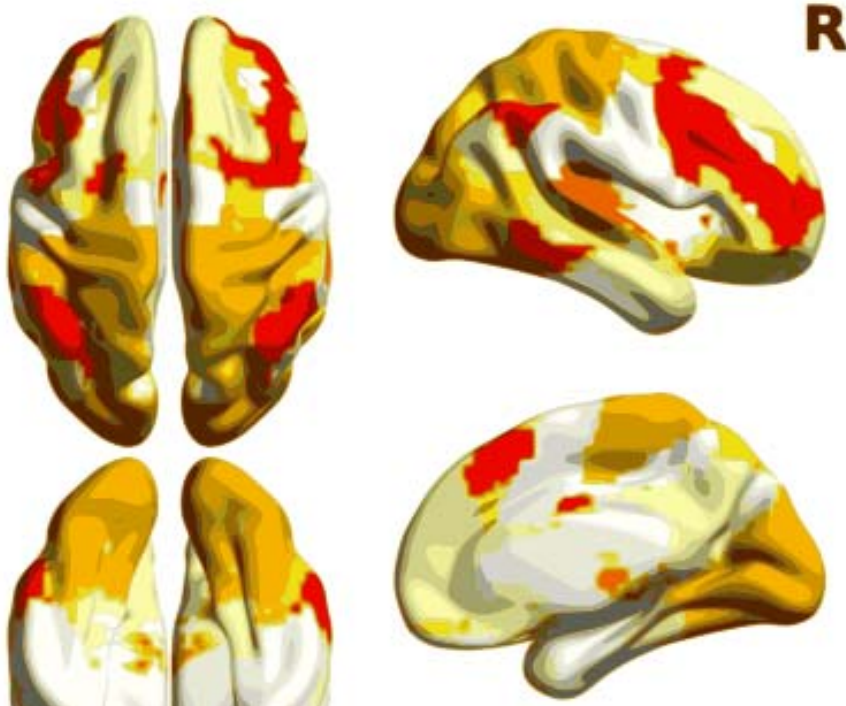

25

\section{PDS effect}

(Likelihood Ratio Test)

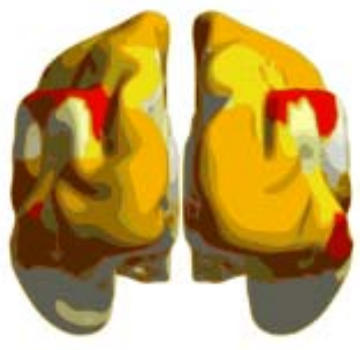

Figure 5. Brain maps of the Likelihood Ratio Test of the GAMM LOESS of the Pubertal Developmental Scale (PDS) term for $B_{0}-A U C$ at the functional networks level. Mapping was based on ROI corresponding to the consensus area according to Power et al. (2011), using BrainNet Viewer (Xia et al., 2013; RRID:SCR_009446). 


\section{Fronto-Parietal Network (B0-AUC)}



Figure 6. Scatter-plot of the GAMM LOESS-PDS model for the $B_{0}-A U C$ residuals (after regressing out in-scanner motion and head-coil) of the Fronto-Parietal Network (FPN) in relation to the pubertal scale (PDS). Thin lines represent individual trajectories; thick black lines represent the sample LOESS curve (with 95\% confidence-interval shadow).

\section{Discussion}

In this study, we have applied Topological Data Analysis (TDA) on the functional connectomes of a longitudinal sample of typically developing children and adolescents. TDA features show a segregated connectivity structure compared to random networks, but even segregated, the brain connectomes exhibit a more dense connectivity pattern within neighbours connections. Furthermore, this topology 
develops in a nonlinear fashion through adolescence, better described by the pubertal status than by chronological age. This nonlinear effect exhibits a faster connectivity of the whole-network and within the Fronto-Parietal Network just after the onset of the pubertal signs.

Regarding the average Betti curves, the sample intercept for the $B_{0}$ curve showed an inverse sigmoid pattern that replicates previous findings in functional connectivity fMRI (Liang and Wang, 2017; Gracia-Tabuenca et al., 2020; Li et al., 2021) and PET (Lee et al., 2012) studies. Furthermore, the average $B_{0}$ curve of the randomized data reached the single component faster than the observed data, evincing a less segregated network. This random pattern was also replicated in another pediatric sample showing the same faster transition to a single component (Gracia-Tabuenca et al., 2020). Concerning the $B_{1}$, both the sample intercept and randomized curves exhibit a bell-shaped curve with an approximate similar filtration value at their maxima, but lower area for the observed data. This evinces a connectivity structure of lower number of holes, or more densely connected at the local level in the real data compared to the random networks. Thus, $\mathrm{B}_{0}$ tells how fast the whole-network goes from isolated to all-connected nodes, while $B_{1}$ reflects how densely connected are those elements already connected. It is noticeable that on average $B_{0}$ tends to reach the single component at filtration values of $\square=0.5$, but at that point $B_{1}$ curves display their peak number of "holes". This implies that these TDA features not only reflect different levels of connectivity structure, but also, they occur at different connectivity strengths.

About the developmental effects of the TDA features, several models were tested to address the area under the $B_{0}$ and $B_{1}$ curves. Nonlinear additive models surpass the goodness of fit of the linear ones, even controlling for the extra number 
of parameters. Specifically, when considering the pubertal status (assessed by the PDS) without its sex interaction is the model that better fits the AUC for both $B_{0}$ and $B_{1}$. Hence, the development of the functional connectome topology better adjusts the pubertal status than chronological age. This is a relevant finding considering that the pubertal status takes into account non-continuous changes as well as more subtle sex effects than a nonlinear age-sex interaction. No previous studies have addressed the adolescent connectome via TDA, but recent studies have shown a better adjustment with the pubertal status for the developmental trends of the functional connectome (based on graph theory; Gracia-Tabuenca et al., 2021) or the frontostriatal functional connectivity (van Duijvenvoorde et al., 2019).

Concerning whole-brain inferences, the $A U C$ for both $B_{0}$ and $B_{1}$, showed an initial increase from PDS level 1 to 2 , but decreased afterwards. In contrast, when focusing on the chronological age, the turning point is approximately at 12 years old, but showing smoother trends compared to PDS. This means a faster transition to the single component for the $\mathrm{B}_{0}$, while a lower rate of geometric holes for the $\mathrm{B}_{1}$ at the end of the adolescence when considering PDS or age. But only $B_{0}-A \cup C$ effects were significant, which evinces that those changes were more prominent at lower filtration values, i.e., edges with higher functional connectivity (or higher integration). Although TDA was not applied in developmental connectomes yet, previous work on brain functional organization during this period has shown increases along age in the functional segregation (Fair et al., 2009; Satterhwaite et al., 2013a; Gu et al., 2015) and integration (Marek et al., 2015). In addition, when considering the PDS, it has been shown that functional centrality, segregation, efficiency, and integration increases at the end of adolescence (Gracia-Tabuenca et al., 2021). All these studies demonstrate the change in configuration of the brain functional organization 
during adolescence. Lastly, when testing the random-intercepts effect within the developmental models, in the additive model with PDS for the $B_{0}-A U C$ its randomeffects showed a strong effect, which shows the relevance of intra-individual trajectories even though of the loss of degrees of freedom.

Likewise, the $B_{0}-A U C$ along PDS effect was stronger in the Fronto-Parietal Network (FPN), showing a similar nonlinear trend as that for the whole brain network. This demonstrates a faster integration of the FPN nodes at the end of the adolescence. The FPN is a key module of the connectome that is involved in the response to high-demanding tasks (Zanto and Gazzaley, 2013) and it is a fundamental system for the consolidation of executive behavior in the adolescent period (Baum et al., 2017; Chai et al., 2017). Other works on functional connectomes have reported an increase of the FPN connectivity along with other attention related systems at the late adolescence (Kwang et al., 2013; Marek et al., 2015; GraciaTabuenca et al., 2021). Previous studies in animal models have shown brain plasticity associated with puberty-related hormonal changes (Sisk \& Foster, 2004). Neuroimaging studies controlling for the age effects, have also revealed structural changes associated with puberty stage in humans, mainly showing decreased gray matter density but increased white matter density in later stages (Peper et al., 2009; Perrin et al., 2009; Bramen et al., 2011; Herting et al., 2012). This work contributes to the emerging evidence that puberty onset greatly influences the development of the brain functional connectivity (van Duijvenvoorde et al., 2019; Gracia-Tabuenca et al., 2021).

Some limitations of this work should be taken into account. We used relatively short scans which may affect the quality of the data, nonetheless it was considered sufficient at the time of the first acquisition (Van Dijk et al., 2010). Furthermore, we 
applied a strident quality control of the data discarding those datasets with less than $80 \%$ good quality data in terms of motion artifact. Nonetheless, the Topological Data Analysis (TDA) complements other network modelling strategies by extracting highdimensional features across the whole range of connectivity values, instead of exploring a fixed set of connections.

\section{Conclusion}

The present study focused on the characterization of functional connectomes as topological spaces in a longitudinal sample of typically developing children and adolescents. Observed Topological Data Analysis (TDA) features showed higher segregation and denser local connections compared to random networks. However, during adolescence this effect changes with a nonlinear trend that increases the integration of the whole-brain and the Fronto-Parietal Network, particularly after the onset of the pubertal signs. These results provide evidence of the nonlinear, pubertydependent developmental trajectories of the topology of the brain network. With the advantage that these properties arise exploring the whole range of connectivity strengths instead of focusing on a small set of them. Being adolescence a critical period for the appearance of the first signs of mental health disorders, we expect these trajectories may be of interest for studying both normal and altered development. 


\section{References}

Akaike, H. (1974). A new look at the statistical model identification. IEEE transactions on automatic control, 19(6), 716-723. https://doi.org/10.1109/TAC.1974.1100705

Avants, B. B., Epstein, C. L., Grossman, M., \& Gee, J. C. (2008). Symmetric diffeomorphic image registration with cross-correlation: evaluating automated labeling of elderly and neurodegenerative brain. Medical image analysis, 12(1), 26-41. https://doi.org/10.1016/j.media.2007.06.004

Bates, D., Sarkar, D., Bates, M. D., \& Matrix, L. (2007). The Ime4 package. R package version, 2(1), 74. https://CRAN.R-project.org/package=Ime4

Baum, G. L., Ciric, R., Roalf, D. R., Betzel, R. F., Moore, T. M., Shinohara, R. T., ... \& Cook, P. A. (2017). Modular segregation of structural brain networks supports the development of executive function in youth. Current Biology, 27(11), 1561-1572. https://doi.org/10.1016/j.cub.2017.04.051

Benjamini, Y., \& Hochberg, Y. (1995). Controlling the false discovery rate: a practical and powerful approach to multiple testing. Journal of the Royal statistical society: series B (Methodological), 57(1), 289-300. https://doi.org/10.1111/i.2517-6161.1995.tb02031.x

Biswal, B., Zerrin Yetkin, F., Haughton, V. M., \& Hyde, J. S. (1995). Functional connectivity in the motor cortex of resting human brain using echo $\square$ planar MRI. Magnetic resonance in medicine, 34(4), 537-541.

\section{https://doi.org/10.1002/mrm.1910340409}

Bramen, J. E., Hranilovich, J. A., Dahl, R. E., Forbes, E. E., Chen, J., Toga, A. W., Dinov, I. D., Worthman, C. M., \& Sowell, E. R. (2011). Puberty influences medial temporal lobe and cortical gray matter maturation differently in boys 
than girls matched for sexual maturity. Cerebral Cortex, 21(3):636-646. https://doi.org/10.1093/cercor/bhq137

Centeno, E. G. Z., Moreni, G., Vriend, C., Douw, L., \& Santos, F. A. (2021). A hands-on tutorial on network and topological neuroscience. bioRxiv. https://doi.org/10.1101/2021.02.15.431255

Chai, L. R., Khambhati, A. N., Ciric, R., Moore, T. M., Gur, R. C., Gur, R. E., ... \& Bassett, D. S. (2017). Evolution of brain network dynamics in neurodevelopment. Network Neuroscience, 1(1), 14-30. https://doi.org/10.1162/NETN a 00001

Ciric, R., Wolf, D. H., Power, J. D., Roalf, D. R., Baum, G. L., Ruparel, K., ... \& Satterthwaite, T. D. (2017). Benchmarking of participant-level confound regression strategies for the control of motion artifact in studies of functional

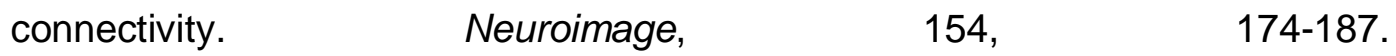
https://doi.org/10.1016/..neuroimage.2017.03.020

Edelsbrunner, H., Letscher, D., \& Zomorodian, A. (2000, November). Topological persistence and simplification. In Proceedings 41st annual symposium on foundations of computer science (pp. 454-463). IEEE. https://doi.org/10.1109/SFCS.2000.892133

Expert, P., Lord, L. D., Kringelbach, M. L., \& Petri, G. (2019). Editorial: Topological neuroscience. Network Neuroscience, 3(3), 653-655. https://doi.org/10.1162/netn e 00096

Fair, D. A., Cohen, A. L., Power, J. D., Dosenbach, N. U., Church, J. A., Miezin, F. M., ... \& Petersen, S. E. (2009). Functional brain networks develop from a "local to distributed" organization. PLoS comput biol, 5(5), e1000381. https://doi.org/10.1371/journal.pcbi.1000381 
Fasy, B. T., Kim, J., Lecci, F., \& Maria, C. (2014). Introduction to the R package TDA. arXiv preprint arXiv:1411.1830. https://arxiv.org/abs/1411.1830

Fonov, V., Evans, A. C., Botteron, K., Almli, C. R., McKinstry, R. C., Collins, D. L., \& Brain Development Cooperative Group. (2011). Unbiased average ageappropriate atlases for pediatric studies. Neuroimage, 54(1), 313-327. https://doi.org/10.1016/..neuroimage.2010.07.033

Giusti, C., Pastalkova, E., Curto, C., \& Itskov, V. (2015). Clique topology reveals intrinsic geometric structure in neural correlations. Proceedings of the National Academy of Sciences, 112(44), 13455-13460. https://doi.org/10.1073/pnas.1506407112

Gracia-Tabuenca, Z., Díaz-Patiño, J. C., Arelio, I., \& Alcauter, S. (2020). Topological Data Analysis reveals robust alterations in the whole-brain and frontal lobe functional connectomes in Attention-Deficit/Hyperactivity Disorder. eNeuro, 7(3). https://doi.org/10.1523/ENEURO.0543-19.2020

Gracia-Tabuenca, Z., Moreno, M. B., Barrios, F. A., \& Alcauter, S. (2021). Development of the brain functional connectome follows puberty-dependent

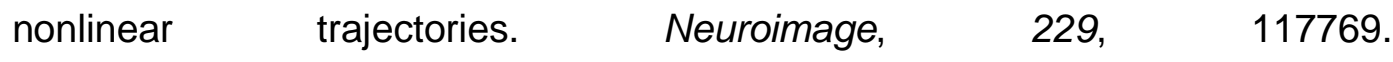
https://doi.org/10.1016/i.neuroimage.2021.117769

Graff, K., Tansey, R., Ip, A., Rohr, C. S., Dimond, D., Dewey, D., \& Bray, S. (2020). Benchmarking common preprocessing strategies in early childhood functional connectivity MRI. bioRxiv. https://doi.org/10.1101/2020.10.27.358192 Gu, S., Satterthwaite, T. D., Medaglia, J. D., Yang, M., Gur, R. E., Gur, R. C., \& Bassett, D. S. (2015). Emergence of system roles in normative neurodevelopment. Proceedings of the National Academy of Sciences, 112(44), 13681-13686. https://doi.org/10.1073/pnas.1502829112 
Herting, M. M., Maxwell, E. C., Irvine, C., \& Nagel, B. J. (2012). The impact of sex, puberty, and hormones on white matter microstructure in adolescents. Cerebral Cortex, 22(9):1979-1992. https://doi.org/10.1093/cercor/bhr246

Hibberd, E. E., Hackney, A. C., Lane, A. R., \& Myers, J. B. (2015). Assessing biological maturity: chronological age and the pubertal development scale predict free testosterone in adolescent males. Journal of Pediatric Endocrinology and Metabolism, 28(3-4), 381-386. https://doi.org/10.1515/jpem$\underline{2014-0187}$

Hwang, K., Hallquist, M. N., \& Luna, B. (2013). The development of hub architecture in the human functional brain network. Cerebral Cortex, 23(10), 2380-2393. https://doi.org/10.1093/cercor/bhs227

Jenkinson, M., Bannister, P., Brady, M., \& Smith, S. (2002). Improved optimization for the robust and accurate linear registration and motion correction of brain images. Neuroimage, 17(2), 825-841. https://doi.org/10.1006/nimg.2002.1132

Jenkinson, M., Beckmann, C. F., Behrens, T. E., Woolrich, M. W., \& Smith, S. M. $\begin{array}{lll}\text { (2012). } & \text { FSL. } & \text { Neuroimage, }\end{array}$ https://doi.org/10.1016/j.neuroimage.2011.09.015

Laube, C., van den Bos, W., \& Fandakova, Y. (2020). The relationship between pubertal hormones and brain plasticity: Implications for cognitive training in adolescence. Developmental Cognitive Neuroscience, 42, 100753. https://doi.org/10.1016/j.den.2020.100753 
Lee, H., Kang, H., Chung, M. K., Kim, B. N., \& Lee, D. S. (2012). Persistent brain network homology from the perspective of dendrogram. IEEE transactions on medical imaging, 31(12), 2267-2277. https://doi.org/10.1109/TMI.2012.2219590

Lee, H., Kang, H., Chung, M. K., Lim, S., Kim, B. N., \& Lee, D. S. (2017). Integrated multimodal network approach to PET and MRI based on multidimensional persistent homology. Human Brain Mapping, 38(3), 1387-1402. https://doi.org/10.1002/hbm.23461

Li, J., Bian, C., Luo, H., Chen, D., Cao, L., \& Liang, H. (2021). Multi-dimensional persistent feature analysis identifies connectivity patterns of resting-state brain networks in Alzheimer's disease. Journal of Neural Engineering. https://doi.org/10.1088/1741-2552/abc7ef

Liang, H., \& Wang, H. (2017). Structure-function network mapping and its assessment via persistent homology. PLoS computational biology, 13(1), e1005325. https://doi.org/10.1371/journal.pcbi.1005325

Manjón, J. V., Coupé, P., Martí $\square$ Bonmatí, L., Collins, D. L., \& Robles, M. (2010). Adaptive non $\square$ local means denoising of MR images with spatially varying noise levels. Journal of Magnetic Resonance Imaging, 31(1), 192-203. https://doi.org/10.1002/jmri.22003

Marek, S., Hwang, K., Foran, W., Hallquist, M. N., \& Luna, B. (2015). The contribution of network organization and integration to the development of cognitive control. PLoS biology, 13(12), e1002328. https://doi.org/10.1371/journal.pbio.1002328

Parkes, L., Fulcher, B., Yücel, M., \& Fornito, A. (2018). An evaluation of the efficacy, reliability, and sensitivity of motion correction strategies for resting-state 
functional MRI. Neuroimage, $\quad$ 171, $\quad$ 415-436. https://doi.org/10.1016/j.neuroimage.2017.12.073

Peper, J. S., Schnack, H. G., Brouwer, R. M., Van Baal, G. C. M., Pjetri, E., Szekely, E., Van Leeu-wen, M., Van Den Berg, S. M., Collins, D. L., Evans, A. C., et al. (2009). Heritability of regional and global brain structure at the onset of puberty: A magnetic resonance imaging study in 9-year-old twin pairs. Human brain mapping, 30(7):2184-2196. https://doi.org/10.1002/hbm.20660

Perrin, J. S., Leonard, G., Perron, M., Pike, G. B., Pitiot, A., Richer, L., Veillette, S., Pausova, Z., \& Paus, T. (2009). Sex differences in the growth of white matter during adolescence. Neuroimage, 45(4):1055-1066. https://doi.org/10.1016/..neuroimage.2009.01.023

Petersen, A. C., Crockett, L., Richards, M., \& Boxer, A. (1988). A self-report measure of pubertal status: Reliability, validity, and initial norms. Journal of youth and adolescence, 17(2), 117-133. https://doi.org/10.1007/BF01537962

Power, J. D., Cohen, A. L., Nelson, S. M., Wig, G. S., Barnes, K. A., Church, J. A., ... \& Petersen, S. E. (2011). Functional network organization of the human brain. Neuron, 72(4), 665-678. https://doi.org/10.1016/..neuron.2011.09.006

Rubinov, M., \& Sporns, O. (2010). Complex network measures of brain connectivity: uses and interpretations. Neuroimage, 52(3), 1059-1069. https://doi.org/10.1016/j.neuroimage.2009.10.003

Santos, F. A., Raposo, E. P., Coutinho-Filho, M. D., Copelli, M., Stam, C. J., \& Douw, L. (2019). Topological phase transitions in functional brain networks. $\begin{array}{llll}\text { Physical Review } & \text { E, } & 100(3), & 032414 .\end{array}$ https://doi.org/10.1103/PhysRevE.100.032414 
Satterthwaite, T. D., Wolf, D. H., Loughead, J., Ruparel, K., Elliott, M. A., Hakonarson, H., ... \& Gur, R. E. (2012). Impact of in-scanner head motion on multiple measures of functional connectivity: relevance for studies of neurodevelopment in youth. Neuroimage, 60(1), 623-632. https://doi.org/10.1016/j.neuroimage.2011.12.063

Satterthwaite, T. D., Wolf, D. H., Ruparel, K., Erus, G., Elliott, M. A., Eickhoff, S. B., ... \& Gur, R. C. (2013a). Heterogeneous impact of motion on fundamental patterns of developmental changes in functional connectivity during youth. Neuroimage, 83, 45-57. https://doi.org/10.1016/..neuroimage.2013.06.045

Satterthwaite, T. D., Elliott, M. A., Gerraty, R. T., Ruparel, K., Loughead, J., Calkins, M. E., ... \& Wolf, D. H. (2013b). An improved framework for confound regression and filtering for control of motion artifact in the preprocessing of resting-state functional connectivity data. Neuroimage, 64, 240-256. https://doi.org/10.1016/..neuroimage.2012.08.052

Sawyer, S. M., Azzopardi, P. S., Wickremarathne, D., \& Patton, G. C. (2018). The age of adolescence. The Lancet Child \& Adolescent Health, 2(3), 223-228. https://doi.org/10.1016/S2352-4642(18)30022-1

Sisk, C. L. \& Foster, D. L. (2004). The neural basis of puberty and adolescence. Nature neuroscience, 7(10):1040-1047. https://doi.org/10.1038/nn1326

Sizemore, A. E., Giusti, C., Kahn, A., Vettel, J. M., Betzel, R. F., \& Bassett, D. S. (2018). Cliques and cavities in the human connectome. Journal of computational neuroscience, 44(1), 115-145. https://doi.org/10.1007/s10827$\underline{017-0672-6}$

Sizemore, A. E., Phillips-Cremins, J. E., Ghrist, R., \& Bassett, D. S. (2019). The importance of the whole: topological data analysis for the network 
neuroscientist. Network Neuroscience, 3(3), 656-673. https://doi.org/10.1162/netn a 00073

Smith, S. M., Fox, P. T., Miller, K. L., Glahn, D. C., Fox, P. M., Mackay, C. E., ... \& Beckmann, C. F. (2009). Correspondence of the brain's functional architecture during activation and rest. Proceedings of the national academy of sciences, 106(31), 13040-13045. https://doi.org/10.1073/pnas.0905267106

Stasinopoulos, D. M., \& Rigby, R. A. (2007). Generalized additive models for location scale and shape (GAMLSS) in R. Journal of Statistical Software, 23(7), 1-46. https://doi.org/10.18637/jss.v023.i07

Tustison, N. J., Avants, B. B., Cook, P. A., Zheng, Y., Egan, A., Yushkevich, P. A., \& Gee, J. C. (2010). N4ITK: improved N3 bias correction. IEEE transactions on medical imaging, 29(6), 1310-1320. https://doi.org/10.1109/TMI.2010.2046908

Van Dijk, K. R., Hedden, T., Venkataraman, A., Evans, K. C., Lazar, S. W., \& Buckner, R. L. (2010). Intrinsic functional connectivity as a tool for human connectomics: theory, properties, and optimization. Journal of neurophysiology, 103(1), 297-321. https://doi.org/10.1152/jn.00783.2009

van Duijvenvoorde, ACK, Westhoff, B, de Vos, F, Wierenga, LM, Crone, EA. A threewave longitudinal study of subcortical-cortical resting-state connectivity in adolescence: Testing age-and puberty-related changes. Human Brain Mapping. 2019; 40: 3769-3783. https://doi.org/10.1002/hbm.24630

Vijayakumar, N., de Macks, Z. O., Shirtcliff, E. A., \& Pfeifer, J. H. (2018). Puberty and the human brain: insights into adolescent development. Neuroscience \& Biobehavioral Reviews, 92 , 417-436. https://doi.org/10.1016/j.neubiorev.2018.06.004 
Wood, S., Scheipl, F., \& Wood, M. S. (2017). Package 'gamm4'. American Statistician, 45, 339. https://CRAN.R-project.org/package=gamm4

Xia, M., Wang, J., \& He, Y. (2013). BrainNet Viewer: a network visualization tool for human brain connectomics. PloS one, 8(7), e68910. https://doi.org/10.1371/journal.pone.0068910

Zanto, T. P., \& Gazzaley, A. (2013). Fronto-parietal network: flexible hub of cognitive control. Trends in cognitive sciences, 17(12), 602-603. https://doi.org/10.1016/j.tics.2013.10.001 